\title{
Trends in binge drinking in Canada from 1996 to 2013: a repeated cross-sectional analysis
}

\author{
Andrew G.M. Bulloch PhD, Jeanne V.A. Williams MSc, Dina H. Lavorato MSc, Scott B. Patten MD PhD
}

Abstract

Background: Heavy drinking is a major factor in morbidity and mortality worldwide. Little information is available on trends in Canada regarding alcohol abuse. We sought to estimate abstinence, binge drinking and alcohol intake exceeding low-risk drinking guidelines in the Canadian population from 1996 to 2013.

Methods: The data sources for this analysis were a series of cross-sectional national health surveys of the Canadian population carried out by Statistics Canada between 1996 and 2013. These were cross-sectional files from the National Population Health Surveys of 1996 and 1998, plus the Canadian Community Health Surveys from 2000 to 2013. Respondents were aged 18 years and older.

Results: The proportion of binge drinkers increased steadily from 13.7\% (95\% confidence interval $[\mathrm{Cl}] 13.2 \%-14.2 \%)$ in 1996 to $19.7 \%$ (95\% Cl 19.1\%-20.3\%) in 2013. The corresponding proportions for men were $20.8 \%(95 \% \mathrm{Cl} 19.9 \%-21.7 \%)$ in 1996 , and $25.7 \%(95 \% \mathrm{Cl} 24.7 \%-26.6 \%)$ in 2013; for women, these proportions were $6.9 \%(95 \% \mathrm{Cl} 6.4 \%-7.5 \%)$ in 1996 , and $13.8 \%(95 \% \mathrm{Cl}$ $13.1 \%-14.5 \%$ ) in 2013. No significant increases were seen in the proportion of people who exceeded low-risk drinking guidelines or of abstainers during the same period.

Interpretation: The rate of self-reported binge drinking in Canada has increased from 1996 to 2013, relatively more so among women than among men. No evidence of an increase in the proportion of people exceeding low-risk drinking guidelines or of abstainers was seen during the same period. These results suggest that binge drinking is of particular concern regarding intervention strategies aimed at improvement of public health.

\section{A} buse of alcohol contributes substantially to morbidity and mortality worldwide. For example, on a global basis, alcohol is estimated to cause $5.2 \%$ of disability adjusted-life years lost because of disease. ${ }^{1}$ When additional causes of deaths are included (e.g., injuries and road accidents) alcohol is estimated to cause $10.0 \%$ of disability adjusted-life years lost and $5.2 \%$ of deaths. ${ }^{2}$ In addition, alcohol abuse is associated with liver cirrhosis, cardiovascular disease, diabetes, psychiatric disorders, cancer and significant social problems, ${ }^{3,4}$ as well as increased risk of suicide ideation and suicide attempts. ${ }^{5,6}$ Intervention strategies at improving public heath require sound epidemiologic data on alcohol use over time, this being the goal of the current study of the Canadian general population.

Binge drinking is known to be a particularly dangerous form of alcohol abuse associated with traffic crashes, sexual violence, homicide and harm to others. ${ }^{7}$ One of the first population estimates of binge drinking was for American college students, ${ }^{8}$ although the definition of binge drinking can be traced back to at least 1969. ${ }^{\text {This }}$ Theasure is not without its critics; for example, blood alcohol content may be a more sensitive measure of problem drinking, ${ }^{10}$ although not a practical approach in epidemiologic surveys.

Previous studies have estimated the prevalence of binge drinking in a number of countries. There has been a tendency to focus on alcohol abuse in children and adolescents ${ }^{11,12}$ and in college students; ${ }^{13}$ however, binge drinking is also an adult problem and can extend into later years. ${ }^{14} \mathrm{~A}$ study of Chinese adults living in Hong Kong reported a 9.0\% prevalence of binge drinking, ${ }^{15}$ whereas a corresponding estimate for Brazil is $11.4 \%,{ }^{16}$ and for countries in Africa it is $9.6 \% .{ }^{17}$ In a large survey of alcohol use in New Zealand, frequent binge drinking was reported by $16.7 \%$ of the population, ${ }^{18}$ and the corresponding proportion for young women in Australia is $16 \% .{ }^{19}$

Competing interests: None declared.

This article has been peer reviewed.

Correspondence to: Andrew Bulloch, bulloch@ucalgary.ca

CMAJ Open 2016. DOI:10.9778/cmajo.20150124 
In the United States, an older study of data from 19 states showed that the prevalence of binge drinking decreased from $16.9 \%$ in 1985 to $13.6 \%$ in $1999 .{ }^{20}$ However, a more recent, large, nationally representative survey of adults in the US found binge drinking to have increased from $21.5 \%$ to $25.8 \%$ between 2001-2002 and 2012-2013. ${ }^{21}$ In all studies, the prevalence of binge drinking is higher among men than among women, and highest in young age groups.

The goal of the present study was to estimate binge and over guideline drinking in the Canadian population by analyzing data from multiple health surveys over the period from 1996 to 2013. Regression was then used to examine trends over time. We compare our results with data from the Canadian Alcohol and Drug Use Monitoring Survey (CADUMS), which has been monitoring alcohol abuse in Canada since 2008.22

\section{Methods}

\section{Sources of data}

This study used the cross-sectional data files collected in 2 early cycles of the National Population Health Survey (1996 and 1998), the general health surveys of the Canadian Community Health Survey (2000, 2003, 2005, 2007/2008, 2009/2010, 2011/2012 and 2013), plus the 2 mental health Canadian Community Health Survey of 2002 and 2012 (Table 1). These surveys used a complex multistage sampling procedure to obtain a representative sample of the Canadian population. First geographical clusters were selected, then households were selected within the clusters. Finally, 1 respondent per household was selected.

\section{Alcohol measures}

Alcohol consumption was measured by estimating several variables in the alcohol module. People who abstained from alcohol were identified based on the results of the question "During the

Table 1: Sample size availability from surveys

\begin{tabular}{|lc|}
\hline Survey & $\begin{array}{c}\text { No. of respondents } \\
(\text { age } \geq 18 \mathrm{yr})\end{array}$ \\
\hline NPHS 1996 & 68282 \\
\hline NPHS 1998 & 14150 \\
\hline CCHS 1.1 (2000) & 118336 \\
\hline CCHS 1.2 (2002) & 35236 \\
\hline CCHS 2.1 (2003) & 121300 \\
\hline CCHS 3.1 (2005) & 120559 \\
\hline CCHS 2007/2008 & 120838 \\
\hline CCHS 2009/2010 & 113796 \\
\hline CCHS 2011/2012 & 115131 \\
\hline CCHS 2012 mental health & 23846 \\
\hline CCHS 2013 & 59224 \\
\hline $\begin{array}{l}\text { Note: CCHS = Canadian Community Health Survey, NPHS = National } \\
\text { Population Health Survey. }\end{array}$ \\
\hline
\end{tabular}

past 12 months, have you had a drink of beer, wine, liquor or any other alcoholic beverage?" For exceeding low-risk drinking guidelines, respondents were asked "On the days you drank in the past 12 months, about how many drinks did you have per day?" The answers were used to construct a 7-day diary of alcohol consumption to identify respondents who exceeded lowrisk drinking guidelines (15 and 10 drinks per week, or 4 and 3 per day, for men and women, respectively). ${ }^{23}$ Finally, binge drinking was assessed with the question "How often in the past 12 months have you had 5 or more drinks on one occasion?" and was defined as consumption of 5 or more drinks at least once a month in the past year, the usual definition of binge drinking used in Statistics Canada surveys.

Although data are available for individuals $12-17$ years old, the data presented here are for people aged 18 years and older from across Canada. This is the legal age for drinking in Alberta, Manitoba and Quebec. In some sensitivity analyses, other age cut-offs and selected provinces were employed.

The CADUMS survey uses its own definitions of at-risk drinking and does not report binge drinking using the 5-drink measure. We used the original data from the public use files to estimate the prevalence of binge drinking in 2008 and 2012.

\section{Statistical analysis}

We initially examined the data with forest plots of frequency estimates with 95\% CIs for each survey. Appropriate sampling weights and bootstrap variance estimation procedures were employed, as recommended by Statistics Canada. Heterogeneity was first examined using the $I^{2}$ statistic, which represents the residual variation due to heterogeneity. Subsequently, randomeffects regression was used to quantify changes over time (i.e., time was a variable included in the models) and to adjust for study level covariates. The decision to use a random-effects model was made a priori. It is a more conservative approach because it does not assume a common distribution. In addition, if the interstudy variability is small ( $\tau^{2}$ approaches 0 ), it becomes increasingly similar and ultimately equivalent to a fixed-effects model. Variables included as potential confounders and effect modifiers included age and sex. Goodness-of-fit was assessed by the associated $I^{2}$ value, the $R^{2}$ value (proportion of betweenstudy variance explained) and the $\tau^{2}$ values (estimates of between-study variance). To test for a possible interaction between sex and time, regression models were run with sex, time and a sex-time interaction term. These analyses used the "metan" command in Stata version $13^{24}$ and were conducted in the Prairie Regional Data Centre of Statistics Canada at the University of Calgary.

\section{Ethics approval}

This research was approved by the Ethics Review Board of the University of Calgary.

\section{Results}

The surveys used in the current study and the total number of available observations are shown in Table 1 . As is typical of Statistics Canada surveys, missing data were minimal (<2\%). 
Inspection of the raw data for binge drinking shows an increase from 1996 to 2013 from 13.7\% (95\% confidence interval [CI $13.2 \%-14.2 \%$ ) to $19.7 \%$ (95\% CI 19.1\%-20.3\%) overall. As expected, the proportion of men engaging in binge drinking was higher than that for women, the increase for men being from $20.8 \%$ (95\% CI $19.9 \%-21.7 \%$ ) to $25.7 \%$ (95\% CI $24.7 \%-26.6 \%)$, whereas as for women it was from $6.9 \%(95 \%$ CI $6.4 \%-7.5 \%$ ) to $13.8 \%$ (95\% CI $13.1 \%-14.5 \%$ ). A preliminary inspection of a forest plot (not shown) gave a visual impression that the proportion of men and women who engaged in binge drinking increased from 1996 to 2013 in a linear fashion, the $I^{2}$ values being $95.2 \%$ and $97.8 \%$, respectively. The linear regression model shown in Figure 1 shows a very good visual fit for both men and women whose fitted lines appear to be parallel, the fit being better for women than for men. This visual impression is supported by a lower $I^{2}$ value of $90.2 \%$ and $83.7 \%$ for men and women, the $R^{2}$ values being $57.6 \%$ and $91.6 \%$ for men and women, and the $\tau^{2}$ values being 0.0002 and 0.00004 for men and women, respectively. Time was significant in the models for both men $(\beta=0.003, t=3.49$, $p=0.007)$ and women $(\beta=0.003, t=8.99, p<0.001)$. It is notable that the slope values ( $\beta$ values) are the same for men and women, which confirms the visual impression that the 2 fitted lines are parallel. Furthermore, in a linear regression model including sex, time and a sex-time interaction term, the latter was found to be nonsignificant $(p=0.593)$. In a sensitivity analysis using ages 18 years and older for Alberta, Manitoba and Quebec, versus 19 years and older for the other provinces, very similar results were seen (data not shown). We also found very similar results when comparing data from British Columbia, the Prairies (Alberta, Saskatchewan and Manitoba), Ontario,
Quebec and the Maritimes (New Brunswick, Prince Edward Island, Nova Scotia and Newfoundland) (data not shown).

Inspection of the raw data and a forest plot (not shown) for exceeding low-risk drinking guidelines showed no clear trend between 1996 and 2013 (Figure 2). The overall proportions of people who exceeded low-risk drinking guidelines in 1996 and 2013 were $11.5 \%$ (95\% CI $11.0 \%-12.0 \%)$ and $10.4 \%$ (95\% CI 10.0\%-10.9\%), respectively. As expected, the proportion of men was greater than that of women; in $2013,11.8 \%(95 \%$ CI $11.1 \%-12.5 \%)$ of men and $6.8 \%(95 \%$ CI, $6.3 \%-7.3 \%$ ) of women exceeded low-risk drinking guidelines. Compared with the binge drinking data, data for exceeding low-risk drinking guidelines showed surprising heterogeneity. For example, note the relatively low values for men in 2007-2008 and 2013 (Figure 2). Also as expected, linear regression models for both men and women showed poor fits, time was not significant, $I^{2}$ values were greater than $97 \%$, and $R^{2}$ values were negative.

The raw data and forest plot (not shown) for abstaining from alcohol from 1996 to 2013 was dissimilar to data for both binge drinking and exceeding low-risk drinking guidelines and suggestive of a curvilinear trend. The prevalence data appear to decrease from 1996 to about 2005-2006, with an increase thereafter (Figure 3). The overall proportions of people abstaining from alcohol in 1996 and 2013 were $22.4 \%$ (95\% CI $21.8 \%-23.1 \%$ ) and $20.3 \%$ (19.7\%-20.9\%), respectively. Abstinence was higher among women. For example, the proportions abstaining in 2013 were 24.6\% (95\% CI $23.7 \%-25.6 \%$ ) for women and $15.8 \%$ (95\% CI $15.0 \%-$ $16.7 \%)$ for men. As expected, linear regression models showed poor fits for both sexes, $I^{2}$ and $R^{2}$ values being greater

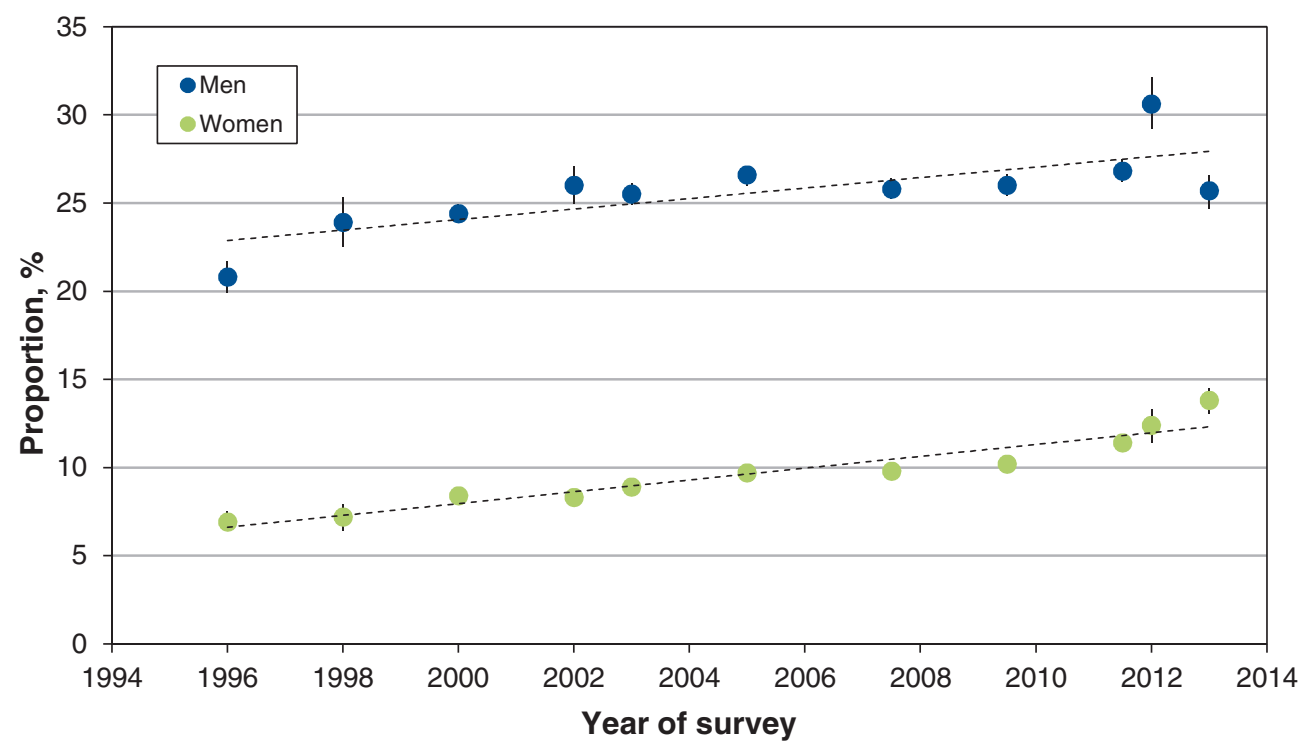

Figure 1: Estimated proportion of binge drinking by sex, 1996-2013. Error bars $=95 \%$ confidence intervals. Dashed lines indicate linear fitted values. 
than $90 \%$ and greater than $20 \%$, respectively. However, addition of a time-squared variable to the model gave a very good visual fit for both sexes (Figure 3). This visual impression is supported by the statistics, with the $I^{2}$ values decreasing to $79.4 \%$ and $86.4 \%$ for men and women, respectively. In addition, the $R^{2}$ values increased to $55.4 \%$ for men and $52.8 \%$ for women, and the $\tau^{2}$ values were 0.0004 and 0.0001 for men and women, respectively. Time was significant in the models for both men $(\beta=-0.005, t=-3.08, p=0.018)$ and women $(\beta=$ $-0.008, t=-2.81, p=0.026)$. Time-squared was significant for men $(\beta=0.0003, t=2.73, p=0.030)$, but was not significant for women $(\beta=0.0003, t=2.32, p=0.053)$.

We used the original data from CADUMS and estimated the prevalence of binge drinking in 2008 and 2012 to be

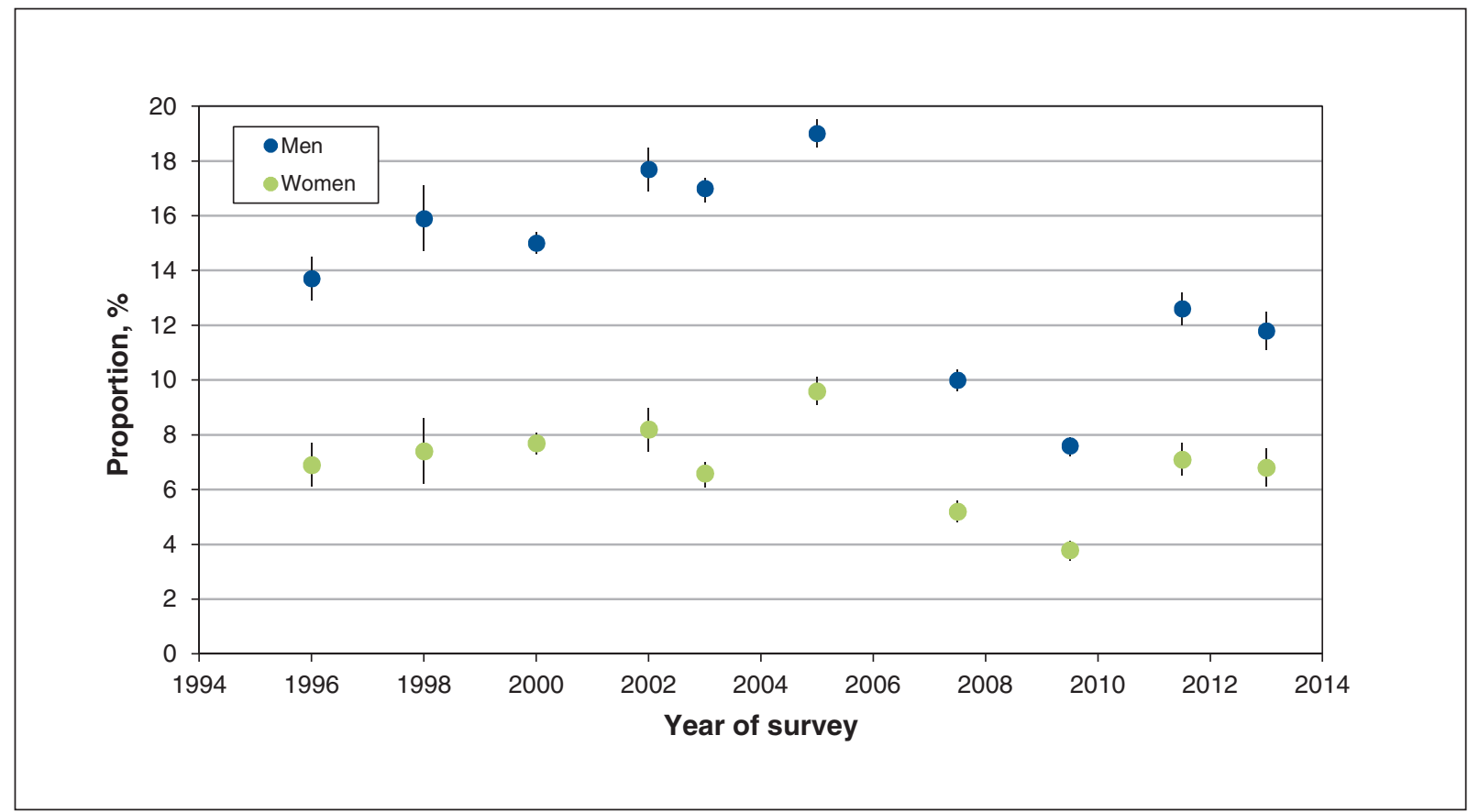

Figure 2: Estimated proportion of exceeding low-risk drinking guidelines, by sex, 1996-2013. Error bars $=95 \%$ confidence intervals.

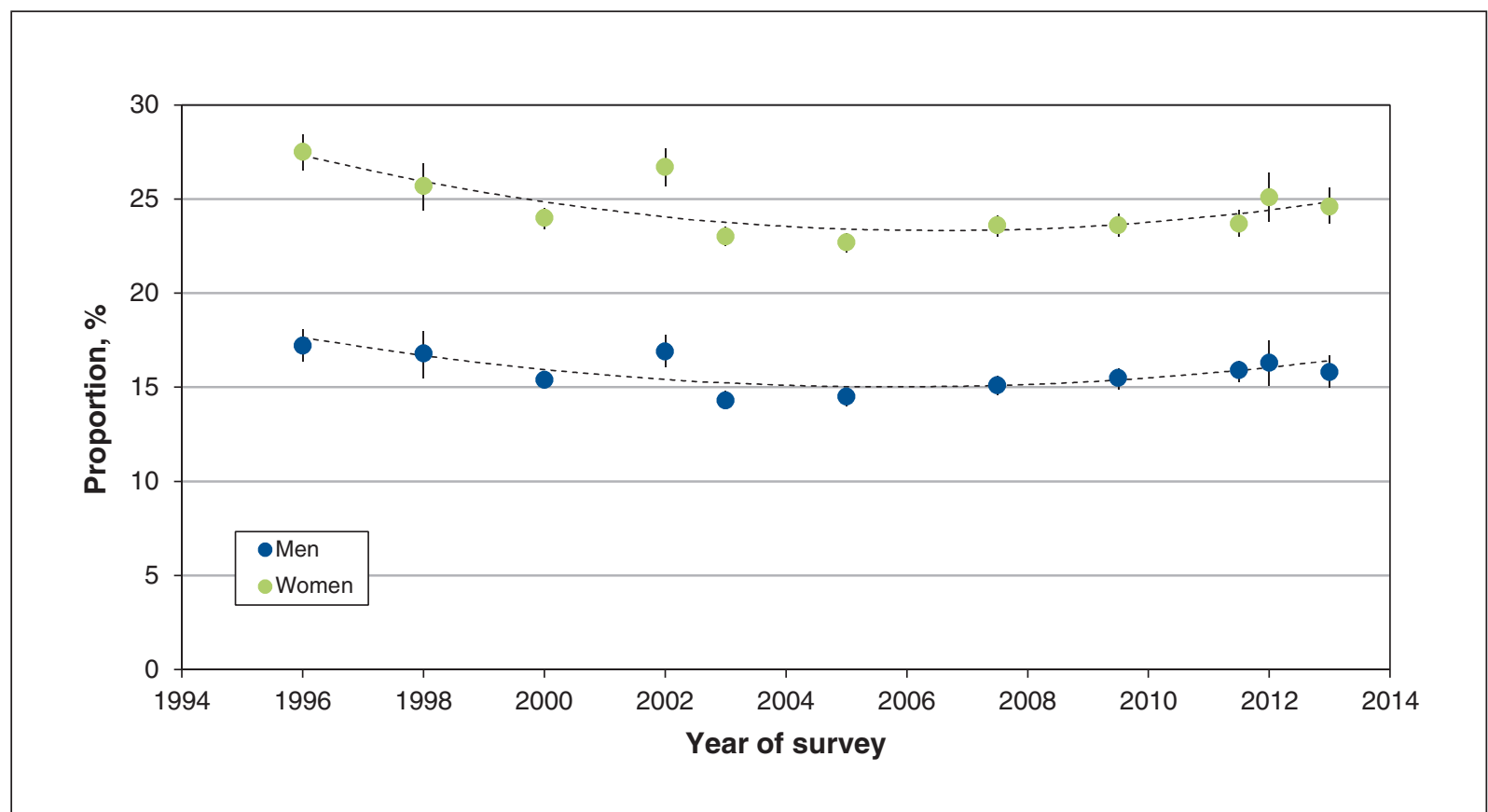

Figure 3: Estimated proportion of abstinence from alcohol, by sex, 1996-2013. Dashed lines indicate quadratic models. Error bars $=95 \%$ confidence intervals. 
16.7\% (95\% CI $15.5 \%-17.9 \%$ ) and $14.8 \%$ (95\% CI $13.6 \%-$ $16.2 \%)$, respectively, with no evidence of a trend during this period. Inspection of our data showed an apparent increase in prevalence in men, but not in women, during the period 2008-2012 (Figure 1).

\section{Interpretation}

A clear linear increase in binge drinking by both men and women was seen from 1996 to 2013 (Figure 1). Visual impressions of these linear trends were confirmed by the statistics accompanying the linear regression analyses, in which time was highly significant. Residual heterogeneity was higher for men than for women (Figure 1). The visual impression that the fitted regression lines are parallel for men and women was confirmed by the slope coefficients ( $\beta$ values), which were the same, and by a statistical test of time-sex interaction. The $I^{2}$ values we report appear to be high. However, the high level of heterogeneity arises from our very precise estimates from large numbers of participants. It has been shown that the heterogeneity statistic, $P$, increases with the number of participants in the analysis and that $\tau^{2}$ is a more appropriate measure. ${ }^{25}$ Here, we present both measures.

In contrast to the binge drinking data, the data for people who exceeded low-risk drinking guidelines were highly heterogeneous and showed no clear trend with time. Attempts to fit linear regression models were unsuccessful, as expected. In terms of abstinence from alcohol, prevalence estimates showed an apparent curvilinear trend, and quadratic equations with a time-squared variable gave very good visual fits; time was significant for both sexes. Time-squared was significant for men $(p=0.030)$, but not for women $(p=0.053)$. Based on these statistics, we conclude there is suggestive, but not conclusive, evidence that favours a quadratic model, with abstinence levels dipping to a low in 2005-2006 and increasing thereafter. To summarize our data, the prevalence of binge drinking has increased steadily from 1996 to 2013, from about $14 \%$ to $20 \%$ overall. The prevalence of exceeding low-risk drinking guidelines was about $6 \%-16 \%$ during this period, with no clear trend over time. Abstinence may have reached a low point in $2005-2006$, and was in the range of $18 \%-22 \%$ from 1996 to 2013.

Our estimates of the prevalence of binge drinking are somewhat lower than 2 estimates for the US that overlap our study period. In 2 large nationally representative surveys of adults in the US, binge drinking was found to have increased from $21.5 \%$ to $25.8 \%$ between $2001-2002$ and $2012-2013 .{ }^{21}$ Our estimates for 2002 and 2013 were $17.0 \%$ and $19.7 \%$, respectively. Given the many similar socioeconomic factors in common between Canada and the US, it would be expected that the extent of binge drinking would be similar in the 2 countries, and the closeness of the estimates helps to reaffirm their probable accuracy. In addition, the increase of binge drinking in the US is apparently part of a longer trend, since earlier estimates for 1991 and 2001 (combined) were in the $14.7 \%-21.6 \%$ range. ${ }^{26}$ Our data are also in line with estimates of increased binge drinking in England from 2001 to 2009, which rose from a prevalence of $21.7 \%$ to $37.9 \%$ during this period. ${ }^{27}$ It should be noted that the United Kingdom definition of binge drinking is 8 (men) or 6 (women) drinks on the heaviest drinking day of the past week. Thus, although direct comparison of binge drinking prevalence in Canada and the US with the UK is difficult, all 3 countries showed a steady increase in overlapping time periods. In contrast to Canada, the US and the UK, estimates of binge drinking prevalence in countries in Africa, in Brazil, and in Hong Kong are much lower, in the $9 \%-12 \%$ range. Whether these lower values are underestimates for these nations is unknown. Whereas our study cannot provide a reason for the increase in binge drinking, a report by the Organization for Economic Cooperation and Development described increased binge drinking by young people and women worldwide and attributes it to alcohol becoming more affordable, more available and more effectively advertised. ${ }^{28}$ It has been observed that binge drinking is subject to age, period and cohort effects, ${ }^{29}$ factors beyond the scope of the present study.

Our results contrast with those we obtained by our analysis of CADUMS data. However, our period of data collection (18 yr) was much longer than that of CADUMS (6 yr), and has the capability to show longer term trends. Another difference between these studies is that the CADUMS population was aged 15 years and older, whereas our population was aged 18 years and older.

\section{Limitations and strengths}

Limitations of the study lie in its reliance on self-report with no independent measures (e.g., blood alcohol concentration). Limitations of the 5-drink definition of binge drinking were discussed in the Introduction. Although the same questions were asked in the same order in all surveys, the position of the questions differed in the 3 types of survey involved, so this could contribute to heterogeneity of the data. An example of heterogeneity is that estimates of exceeding low-risk drinking guidelines among men in 2007/2008 and 2013 were low and close to those among women. We could not explain this heterogeneity based on the provinces sampled, the mode of question (telephone v. in-person) or the position of the questions in the 2 surveys (data not shown), but this supports our use of random-effects models. In addition, our lack of data for youth is a limitation.

We were able to examine drinking trends over an unusually long study period of 18 years (1996-2013). This was made possible by analysis of 11 Statistics Canada health surveys during this period. Another strength is the large sample sizes (ranging from about 14000 to 120000 over the 11 surveys). Furthermore, the surveys used consistent questions to determine alcohol use.

\section{Conclusions and implications for practice and future research}

That one-fifth of the Canadian population currently engages in binge drinking is of considerable concern for public health and provides data for evidence-based health regulations and policies. Binge drinking is known to be a particularly danger- 
ous form of alcohol abuse that is strongly related to myocardial infarction, unsafe sex, violence and injuries. ${ }^{7}$ Furthermore, studies in Canada for 1950-1998 have shown a relationship between alcohol consumption and suicide, allcause mortality and fatal accidents. ${ }^{30-32}$ A number of focused interventions and public health policies have been implemented, ${ }^{3}$ but the observed steady increase in binge drinking poses a major challenge for current and future public health provision. Future research is needed to evaluate the effectiveness of interventions.

\section{References}

1. GBD 2013 DALYs and HALE Collaborators. Murray CJ, Barber RM, Foreman $\mathrm{KJ}$, et al. Global, regional, and national disability-adjusted life years (DALYs) for 306 diseases and injuries and healthy life expectancy (HALE) for 188 countries: quantifying the epidemiological transition. Lancet 2015;386:2145-91.

2. GBD 2013 Risk Factor Collaborators. Global, regional, and national comparative risk assessment of 79 behavioural, environmental and occupational, and metabolic risks or clusters of risks in 188 countries, 1990-2013: a systematic analysis for the Global Burden of Disease study 2013. Lancet 2015;386:2287-323.

3. Giesbrecht N, Stockwell T, Kendall P, et al. Alcohol in Canada: reducing the toll through focused interventions and public health policies. CMA7 2011;183:450-5.

4. Kraus L, Baumeister SE, Pabst A, et al. Association of average daily alcohol consumption, binge drinking and alcohol-related social problems: results from the German Epidemiological Surveys of Substance Abuse. Alcobol Alcohol 2009; $44: 314-320$.

5. Glasheen C, Pemberto MR, Lipari R, et al. Binge drinking and the risk of suicidal thoughts, plans, and attempts. Addict Behav 2015;43:42-9.

6. Schaffer M, Jegli EL, Stanley B. Suicidal behavior, ideation, and binge drinking among college students. Arch Suicide Res 2008;12:124-32.

7. Flegel K, MacDonald N, Hebert PC. Binge drinking: all too prevalent and hazardous [editorial]. CMA7 2011;183:411.

8. Wechsler H, Davenport A, Dowdall G, et al. Health and behavioral consequences of binge drinking in college. a national survey of students at 140 campuses. FAMA 1994;272:1672-7.

9. Wechsler H, Austin SB. Binge drinking: the five/four measure. 7 Stud Alcobol 1998;59:122-4

10. Fillmore MT, Jude MJ. Defining "binge" drinking as five drinks per occasion or drinking to a. $08 \%$ BAC: Which is more sensitive to risk? Am 7 Addict 2011;20:468-75.

11. Wellman RJ, Contreras GA, Dugas EN, et al. Determinants of sustained binge drinking in young adults. Alcobol Clin Exp Res 2014;38:1409-15.

12. Pedersen $W$, von Soest $T$. Adolescent alcohol use and binge drinking: an 18-year trend study of prevalence and causes. Alcobol Alcobol 2015;50:219-25.

13. Beets MW, Flay BR, Vuchinich S, et al. Longitudinal patterns of binge drinking among first year college students with a history of tobacco use. Drug Alcobol Depend 2009;103:1-8.

14. Blazer DG, Wu LT. The epidemiology of at-risk and binge drinking among middle-aged and elderly community adults: national survey on drug use and health. Am 7 Psychiatry 2009;166:1162-9.

15. Kim JH, Lee S, Chow J, et al. Prevalence and the factors associated with binge drinking, alcohol abuse, and alcohol dependence: a population-based study of Chinese adults in Hong Kong. Alcohol Alcohol 2008;43:360-70.

16. Castro DS, Sanchez ZM, Zaleski M, et al. Sociodemographic characteristics associated with binge drinking among Brazilians. Drug Alcobol Depend 2012; 126:272-6.

17. Peltzer K, Davids A, Njuho P. Alcohol use and problem drinking in South Africa: findings from a national population-based study. Afr $\mathcal{F}$ Psychiatry (7ohannesbg) 2011;14:30-7.
18. Jatrana S, Carter K, McKenzie S, et al. Binge drinking is patterned by demographic and socioeconomic position in New Zealand: largest national survey to date. NZ Med F 2011;124:110-5.

19. Powers J, Duffy L, Burns L, et al. Binge drinking and subsequent depressive symptoms in young women in Australia. Drug Alcohol Depend 2016;161:86-94.

20. Serdula MK, Brewer RD, Gillespie C, et al. Trends in alcohol use and binge drinking, 1985-1999. Results of a multi-state study. Am 7 Prev Med 2004; 26:294-8.

21. Dawson DA, Goldstein RB, Saha TD, et al. Changes in alcohol consumption: United States, 2001-2002 to 2012-2013. Drug Alcohol Depend 2015;148:56-61.

22. Canadian Alcohol and Drug Use Monitoring Survey: summary of results for 2012. Ottawa: Health Canada; modified 2014. Available: www.hc-sc.gc.ca/hc-ps/ drugs-drogues/stat/2012/summary-sommaire-eng.php (accessed 2016 Sept. 8).

23. Canada's low-risk alcohol drinking guidelines. Ottawa: Canadian Centre on Substance Abuse; 2011. Available: www.ccsa.ca/Resource\%20Library/2012-Canada-Low -Risk-Alcohol-Drinking-Guidelines-Brochure-en-pdf (accessed 2016 Apr. 24).

24. Stata Statistical Software: Release 13. College Station (TX): StataCorp LP; 2013.

25. Rücker G, Schwarzer G, Carpenter JR, et al. Undue reliance on $I^{2}$ in assessing heterogeneity may mislead. BMC Med Res Methodol 2008;8:79-88.

26. Miller JW, Gfroerer JC, Brewer RD, et al. Prevalence of adult binge drinking. A comparison of two national surveys. Am 7 Prev Med 2004;27:197-204.

27. Twigg L, Moon G. The spatial and temporal development of binge drinking in England 2001-2009: an observational study. Soc Sci Med 2013;91:162-7.

28. Tackling harmful alcohol use. Paris: Organisation for Economic Co-operation and Development; 2015. Available: www.oecd.org/health/tackling-harmful -alcohol-use-9789264181069-en.htm (accessed 2016 July 3).

29. Keyes KM, Miech R. Age, period, and cohort effects in heavy episodic drinking in the US from 1985 to 2009. Drug Alcohol Depend 2013;132:140-8.

30. Ramstedt M. Alcohol and suicide at the population level - the Canadian experience. Drug Alcohol Rev 2005;24:203-8.

31. Norström T. Per capita alcohol consumption and all-cause mortality in Canada, 1950-1998. Addiction 2004;99:1274-8.

32. Skog OJ. Alcohol consumption and fatal accidents in Canada, 1950-1998. Addiction 2003;98:883-93.

Affiliations: Department of Community Health Sciences (Bulloch, Williams, Lavorato, Patten); Department of Psychiatry (Bulloch, Patten; Mathison Centre for Mental Health Research \& Education, Hotchkiss Brain Institute (Bulloch, Patten), University of Calgary, Calgary, Alta.

Contributors: Jeanne Williams and Dina Lavorato conducted the statistical analyses. The first draft of the paper was written by Andrew Bulloch. Scott Patten, Jeanne Williams and Dina Lavorato provided critical feedback on drafts of the paper. Scott Patten was the primary investigator, and Andrew Bulloch was co-investigator, on the grant from Canadian Institutes of Health Research. All authors have read and approved the final version of the paper and have agreed to act as guarantors of the work.

Funding: This project was supported by a grant from the Canadian Institutes of Health Research (CIHR). CIHR had no role in study design, in the collection, analysis and interpretation of data, in the writing of the report, nor in the decision to submit the article for publication. Scott Patten was supported by salary awards from Alberta Innovates-Health Solutions.

Disclaimer: The authors have no conflicts of interest regarding the integrity of the reported findings. This research and analysis were based on data from Statistics Canada, but the opinions expressed do not represent the views of Statistics Canada.

Supplemental information: For reviewer comments and the original submission of this manuscript, please see www.cmajopen.ca/content/4/4/ E599/suppl/DC1 